\title{
ACHADOS NA TRIAGEM IMITANCIOMÉTRICA E DE PROCESSAMENTO AUDITIVO EM ESCOLARES
}

\author{
Acoustic immitance and auditory processing screening \\ findings in school children
}

\author{
Camila Lucia Etges ${ }^{(1)}$, Mariana Citton Padilha dos Reis ${ }^{(2)}$, Isabela Hoffmeister Menegotto (3), \\ Pricila Sleifer ${ }^{(4)}$, Cristina Loureiro Chaves Soldera ${ }^{(5)}$
}

\begin{abstract}
RESUMO
Objetivos: verificar os achados da triagem imitanciométrica e dos testes da avaliação simplificada de processamento auditivo em escolares. Método: participaram da pesquisa alunos de $1^{\underline{a}}$ a $4^{\underline{a}}$ séries, de sete a dez anos de idade, de uma escola de ensino público de Porto Alegre. Foram avaliados 130 escolares na triagem imitanciométrica, que foi constituída por timpanometria e pesquisa do reflexo acústico ipsilateral e avaliação simplificada do processamento auditivo, incluindo testes de localização sonora, memória sequencial para sons verbais e memória sequencial para sons não verbais. Resultados: na triagem imitanciométrica $43,08 \%$ dos escolares passaram, tendo a curva tipo A como mais frequente. O reflexo acústico em $4000 \mathrm{~Hz}$ teve percentual de presença inferior comparado com os demais. Passaram nos testes da avaliação simplificada do processamento auditivo $76,15 \%$ das crianças. Além disso, foi observado que o teste no qual os escolares obtiveram pior desempenho foi o de memória sequencial para sons verbais. Falharam na triagem imitanciométrica e na avaliação simplificada de processamento auditivo $12,3 \%$ dos escolares. Conclusão: a curva timpanométrica tipo A foi a mais frequente na população estudada. Na avaliação simplificada do processamento auditivo a maioria dos sujeitos passou, tendo maior frequência de acertos no teste de localização sonora. Não houve associação estatística entre o resultado da triagem imitanciométrica e o resultado da avaliação simplificada de processamento auditivo.
\end{abstract}

DESCRITORES: Audição; Saúde Escolar; Percepção Auditiva

(1) Fonoaudióloga Graduada pela Universidade Federal de Ciências da Saúde de Porto Alegre (UFCSPA), Porto Alegre, Rio Grande do Sul, Brasil.

(2) Fonoaudióloga Graduada pela Universidade Federal de Ciências da Saúde de Porto Alegre (UFCSPA), Porto Alegre, Rio Grande do Sul, Brasil.

(3) Fonoaudióloga; Professora Adjunto do Curso de Fonoaudiologia da Universidade Federal de Ciências da Saúde de Porto Alegre - UFCSPA; Doutora em Distúrbios da Comunicação Humana pela UNIFESP-EPM, Porto Alegre, Rio Grande do Sul, Brasil.

(4) Fonoaudióloga; Professora Adjunto do Curso de Fonoaudiologia da Universidade Federal do Rio Grande do Sul - UFRGS; Doutora em Ciências Médicas: Pediatria pela UFRGS, Porto Alegre, Rio Grande do Sul, Brasil.

(5) Fonoaudióloga; Professora Assistente do Curso de Fonoaudiologia da Universidade Federal de Ciências da Saúde de Porto Alegre - UFCSPA; Mestre em Distúrbios da Comunicação Humana pela UFSM, Porto Alegre, Rio Grande do Sul, Brasil.

Conflito de interesses: inexistente

\section{INTRODUÇÃO}

A audição é um dos sentidos mais importantes para o aprendizado. Sua integridade depende do funcionamento adequado dos sistemas da orelha externa, média e interna. O seu desenvolvimento está diretamente ligado ao da fala e ao aprendizado de leitura e escrita.

A atuação da Fonoaudiologia na escola tem como objetivos a prevenção e a detecção - por meio de triagens - dos distúrbios da comunicação, como também oferecer orientações aos pais, professores e demais integrantes da equipe escolar ${ }^{1}$. Ao compartilhar com os professores conhecimentos relacionados à prevenção e desenvolvimento da audição, bem como consequências da perda auditiva nas crianças, proporciona-se uma série de benefícios ao ambiente escolar². 
Nas escolas, os testes mais utilizados na triagem auditiva são as medidas de imitância acústica ${ }^{3}$, que fornecem resultados rápidos e eficientes sobre a integridade e o funcionamento do sistema da orelha média. A imitanciometria é um excelente teste diagnóstico, com $85 \%$ de especificidade nos casos de secreção de orelha média, quando demonstra um aumento da impedância na propagação sonora pelo conjunto tímpano-ossicular ${ }^{4}$. Estas medidas são importantes devido à alta incidência de problemas de orelha média em crianças, entre elas a disfunção tubária e a otite média e suas variações ${ }^{5}$. Como consequência da otite média, a criança poderá desenvolver uma perda auditiva do tipo condutiva, geralmente de leve a moderada, que pode resultar em baixo rendimento escolar. Além disso, a criança pode ser rotulada como distraída, pedir para repetir o que os outros falam e ouvir televisão em volume forte ${ }^{6}$.

A privação sensorial decorrente da presença de secreção na orelha média, agravada pelo número e pela duração dos episódios da doença, pode afetar a percepção da fala e dificultar a compreensão, principalmente em ambiente ruidoso, prejudicando o desenvolvimento da linguagem da criança, do aprendizado escolar e do processamento auditivo?.

Os indivíduos que apresentam disfunção do processamento auditivo (DPA) podem apresentar dificuldade de comunicar-se em ambientes ruidosos, de compreender piadas ou palavras de "duplo sentido", atenção reduzida, problemas de memória, dificuldade de entender o que leem, de contar uma história ou dar um recado, dificuldades escolares, principalmente com Português e Matemática, inversão de letras na escrita, dificuldade na produção de determinados sons da fala, como nos fonemas $/ \mathrm{r} / \mathrm{e} / \mathrm{I} /$, enquanto apresentam nível de inteligência normal ${ }^{8}$.

Alguns autores acreditam que a privação sensorial devido à otite média não é capaz de provocar alterações de processamento auditivo ${ }^{9,10}$, porém, em outros estudos, crianças com histórico de otite média nos primeiros anos de vida apresentaram questões de processamento auditivo alteradas ${ }^{11,12}$.

No processamento auditivo, não basta o sujeito possuir limiares auditivos normais, é preciso que o sinal acústico seja analisado e interpretado, para que se transforme em uma mensagem com significado. A perda auditiva não é um fator determinante para alterações de processamento auditivo, pois há indivíduos que mesmo apresentando perda auditiva periférica (de grau leve a moderado), apresentam processamento auditivo normal ${ }^{13}$.

Associada à otite média, a redução na condução da onda sonora, a perda auditiva flutuante e a privação auditiva podem levar ao déficit no processamento auditivo ${ }^{14-16}$. Um estudo apontou que crianças que tiveram otite média no primeiro ano de vida apresentaram maior dificuldade em reconhecer palavras na existência de ruído do que as que não tiveram otite média naquele período ${ }^{11}$.

Sendo assim, o objetivo desta pesquisa foi verificar os achados da triagem imitanciométrica e dos testes da avaliação simplificada do processamento auditivo aplicados em escolares do ensino fundamental.

\section{MÉTODO}

A presente pesquisa trata-se de um estudo transversal realizado em uma escola de ensino público de Porto Alegre, contando com a participação de alunos de $1^{\underline{a}}$ a $4^{\underline{a}}$ série do ensino fundamental. A 1 $1^{a}$, a $2^{\mathrm{a}}$ e a $4^{\mathrm{a}}$ série da escola possuíam três turmas, e a $3^{\text {a }}$ série era composta por duas turmas, totalizando 259 estudantes. As atividades foram desenvolvidas no período de junho a setembro de 2010.

Inicialmente as pesquisadoras foram às salas de aula das séries incluídas na pesquisa e, após autorização da professora responsável pela turma, entregaram dois Termos de Consentimento Livre e Esclarecido (TCLE) para cada aluno, sendo um destes destinado aos pais e outro, elaborado com linguagem mais simples, específico para o consentimento das crianças - conforme solicitação do Comitê de Ética da Universidade. Ambos os termos continham informações sobre os procedimentos a serem realizados, formalizando o aceite da participação da criança no estudo. Não participaram da pesquisa crianças que não tiveram um ou ambos TCLEs assinados.

Foram considerados como critérios de exclusão a criança ter menos de sete anos de idade; apresentar alguma impossibilidade para a realização do exame imitanciométrico, como excesso de cerúmen no conduto auditivo externo ou a não colaboração durante a triagem; ter os termos de consentimento não assinados pelos pais/responsáveis e/ou não ter concordância da criança em participar da pesquisa.

Para a realização das triagens as crianças foram retiradas da sala de aula com consentimento prévio da professora responsável, sendo levadas para uma sala silenciosa, disponibilizada pela escola. A triagem auditiva foi composta por meatoscospia, medidas de imitância acústica (MIA) e avaliação simplificada do processamento auditivo.

A meatoscopia foi realizada com o uso de otoscópio marca WelchAllyn modelo Pocket Junior, para verificar a presença de excesso de cerúmen ou qualquer outro fator que impedisse a realização das avaliações. 
Para a realização das MIA (curva timpanométrica e reflexos acústicos ipsilaterais) foi utilizado o imitanciômetro marca Interacoustics, modelo MT10, com tom de prova de $226 \mathrm{~Hz}$. Considerou-se curva timpanométrica tipo $\mathrm{A}$ quando o pico de máxima admitância esteve ao redor da pressão de ar de $0 \mathrm{daPa}$, cuja variação não exedeu a $-100 \mathrm{daPa}$; curva tipo $\mathrm{B}$ quando não ocorreu o pico de máxima admitância em nenhuma pressão de ar; curva timpanométrica tipo $\mathrm{C}$ quando o pico de máxima admitância esteve deslocado para pressões negativas, abaixo de -100 daPA; curva tipo As quando ocorreu baixa admitância; e curva timpanométrica tipo Ad quando o intervalo entre os dois ramos da curva foi igual ou superior a $100 \mathrm{daPa}^{17}$. O reflexo acústico pesquisado foi do tipo ipsilateral, visto que o equipamento utilizado avalia somente este tipo de reflexo acústico, para as frequências de 500, 1000, 2000 e 4000 Hz. Considerou-se como critério de "passa" desta etapa da triagem a criança que apresentou curva timpanométrica tipo $\mathrm{A}^{18} \mathrm{e}$ reflexos acústicos ipsilaterais presentes em todas as frequências pesquisadas ${ }^{3}$.

Os testes especiais para triagem do processamento auditivo aplicados foram: Localização Sonora em cinco direções (LS), Memória Sequencial para Sons Verbais (MSSV) e Memória Sequencial para Sons Não Verbais (MSSNV) ${ }^{8}$. Os testes de MSSV e MSSNV visam avaliar a habilidade auditiva de memória para sons em sequência ou ordenação temporal.

O teste de LS foi aplicado utilizando-se o guizo, com o objetivo de avaliar a habilidade auditiva de localização sonora. Foram avaliadas cinco direções em relação à cabeça da criança: à frente, atrás, acima, à direita e à esquerda. A instrução foi dada por demonstração, sendo que a criança foi orientada a fechar os olhos e apontar para a direção da qual ele acreditava provir o som. $O$ critério de normalidade para o teste de LS foi de a criança acertar pelo menos quatro das cinco direções apresentadas, sendo que o erro esperado estava em uma das seguintes direções: ou à frente, ou atrás, ou acima da cabeça. Para a pesquisa dos sons verbais foi utilizada a emissão das sílabas "pa", "ta", "ca", e "fa" em três ordens diferentes e a criança tinha que repetir de forma adequada a sequência dos sons. Para fins de análise dos dados, estabeleceu-se sequência 1 para $\mathrm{pa} / \mathrm{ta} / \mathrm{ca} / \mathrm{fa}$, sequência 2 para ta/pa/ca/fa e sequência 3 para ca/fa/ta/pa. Para os sons não verbais foram utilizados quatro instrumentos sonoros - sino, agogô, coco, guizo apresentados em três sequências diferentes, sendo guizo/coco/sino/agogô a sequência $A$, coco/guizo/ sino/agogô a sequência $B$ e sino/guizo/agogô/coco a sequência $\mathrm{C}$. A criança foi orientada a apontar os objetos na ordem em que foram percutidos, sendo que a instrução foi dada por demonstração. Para os testes de MSSV e MSSNV considerou-se como critério de "passa" a criança acertar duas das três sequências sonoras apresentadas ${ }^{8}$. Considerou-se que a criança "passou" na triagem de processamento auditivo quando apresentou respostas dentro do esperado nos três testes aplicados.

Após a realização das triagens a criança recebeu um protocolo de resultado das avaliações para ser entregue aos pais/responsáveis acompanhado de dois folders informativos sobre cuidados com a audição - um direcionado aos pais e outro à criança - elaborado pelo Projeto de Extensão Ouça Bem, do curso de Fonoaudiologia da Universidade Federal de Ciências da Saúde de Porto Alegre.

No protocolo de resultado daquelas crianças que apresentaram falha em uma ou em ambas as triagens, sugeriu-se a realização de consulta médica para verificar a necessidade de encaminhamento para uma avaliação mais completa da audição.

As triagens imitanciométrica e de processamento auditivo não foram necessariamente realizadas no mesmo dia em função da disponibilidade da equipe do Projeto de Extensão Ouça Bem que colaborou na coleta dos dados da pesquisa.

Após o término das triagens foi realizada palestra informativa em reunião de pais e professores da escola para esclarecimento de dúvidas com relação aos resultados das avaliações.

Esta pesquisa foi aprovada pelo Comitê de Ética da Universidade Federal de Ciências da Saúde de Porto Alegre, sob o protocolo número 627/10.

Para análise dos resultados foi utilizado o teste qui-quadrado e o nível de significância adotado foi de 0,05 ou $5 \%$.

\section{RESULTADOS}

Foram avaliados 130 escolares na triagem imitanciométrica e na avaliação simplificada do processamento auditivo. As crianças avaliadas tinham entre sete e 10 anos de idade (Figura 1), distribuídas de $1^{\underline{a}}$ a $4^{a}$ séries do ensino fundamental (Figura 2) - correspondendo a 50,19\% do total de estudantes destas séries.

A figura 3 e a tabela 1 apresentam os resultados da triagem imitanciométrica dos escolares. $\mathrm{Na}$ triagem imitanciométrica, $56(43,08 \%)$ passaram e 74 (56,92\%) falharam.

Das crianças avaliadas no teste de LS, 76 $(58,46 \%)$ fizeram cinco acertos, 45 (34,62\%) quatro acertos e $9(6,92 \%)$ três acertos, sendo que nenhuma fez menos de três acertos. A tabela 2 apresenta os achados no teste de LS. 


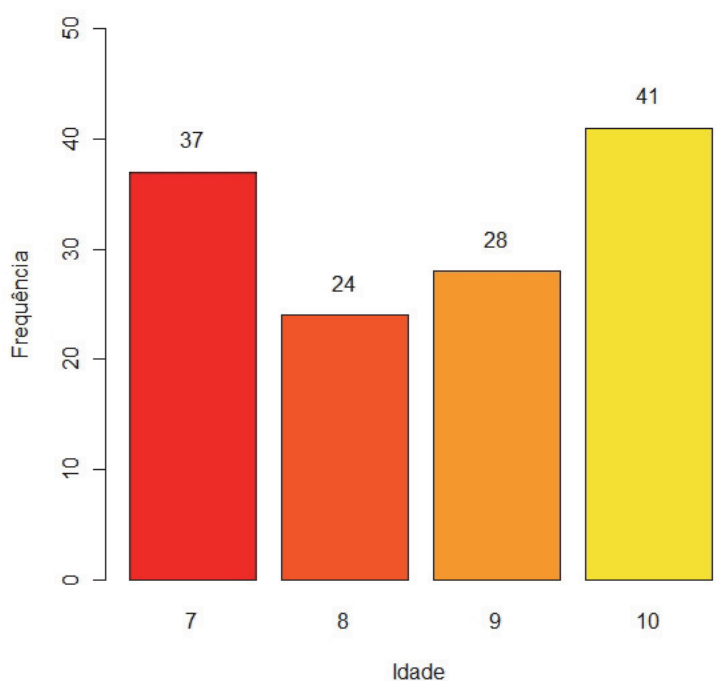

Figura 1 - Distribuição dos escolares avaliados de acordo com a idade

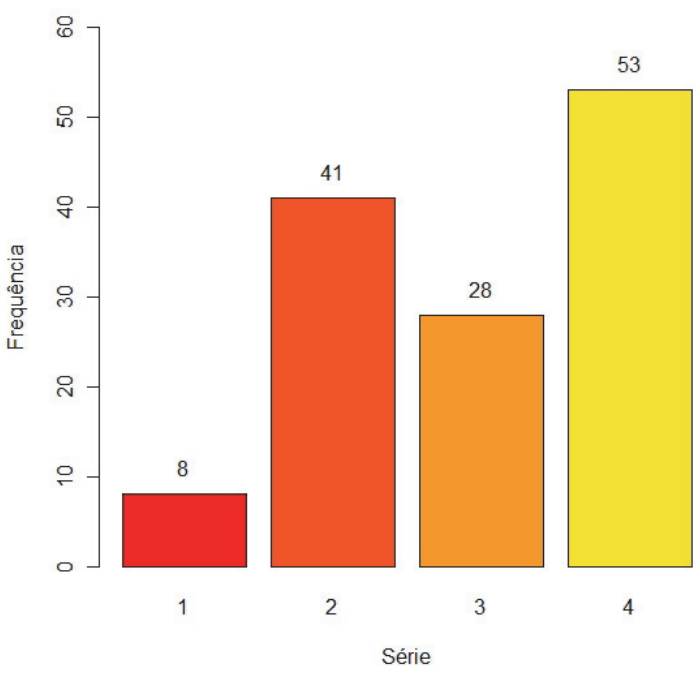

Figura 2 - Distribuição dos escolares avaliados de acordo com a série

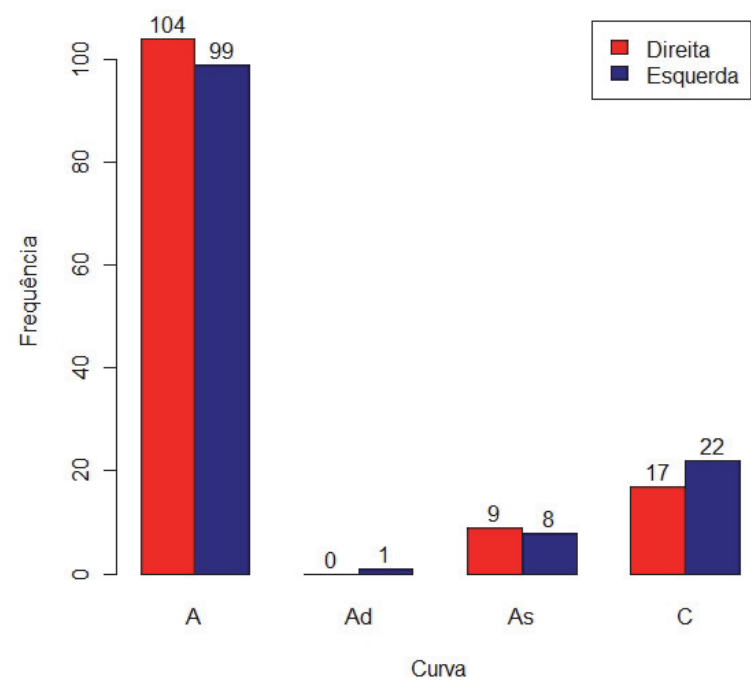

Figura 3 - Tipos de curvas timpanométricas (Jerger) referentes às orelhas direita e esquerda dos escolares avaliados 
Tabela 1 - Ocorrência de reflexos ipsilaterais presentes (por frequência) da população estudada nas orelhas direita e esquerda $(n=130)$

\begin{tabular}{cccccc}
\hline \multirow{2}{*}{ Orelha } & & \multicolumn{4}{c}{ Reflexo } \\
\cline { 2 - 5 } & & $\mathbf{5 0 0 ~ H z}$ & $\mathbf{1 0 0 0 ~ H z}$ & $\mathbf{2 0 0 0 ~ H z}$ & $\mathbf{4 0 0 0 ~} \mathbf{~ z z}$ \\
\hline \multirow{2}{*}{ Direita } & $\mathrm{n}$ & 112 & 106 & 105 & 89 \\
& $\%$ & 86,15 & 81,54 & 80,77 & 68,46 \\
\hline \multirow{2}{*}{ Esquerda } & $\mathrm{n}$ & 109 & 111 & 111 & 93 \\
& $\%$ & 83,85 & 85,38 & 85,38 & 71,54 \\
\hline
\end{tabular}

Tabela 2 - Resultados no teste de Localização Sonora

\begin{tabular}{cccccccc}
\hline \multirow{2}{*}{$\begin{array}{c}\text { Localização } \\
\text { Sonora }\end{array}$} & \multirow{2}{*}{ Acertos } & \multicolumn{5}{c}{ Direção apontada pelos estudantes que erraram a } \\
\cline { 4 - 8 } & & & Direita & Esquerda & Atrás & Frente & Acima \\
\cline { 4 - 8 } Direita & $\mathrm{n}$ & 130 & - & - & - & - & - \\
& $\%$ & 100,00 & - & - & - & - & - \\
\hline \multirow{2}{*}{ Esquerda } & $\mathrm{n}$ & 130 & - & - & - & - & - \\
& $\%$ & 100,00 & - & - & - & - & - \\
\hline \multirow{2}{*}{ Atrás } & $\mathrm{n}$ & 112 & - & 2 & - & 8 & 8 \\
& $\%$ & 86,15 & - & 11,11 & - & 44,44 & 44,44 \\
\hline \multirow{2}{*}{ Frente } & $\mathrm{n}$ & 121 & 3 & 2 & - & - & 3 \\
& $\%$ & 93,08 & 37,50 & 25,00 & - & - & 37,50 \\
\hline \multirow{2}{*}{ Acima } & $\mathrm{n}$ & 93 & - & 6 & 20 & - & 6 \\
& $\%$ & 71,54 & - & 18,75 & 62,50 & - & 18,75 \\
\hline
\end{tabular}

Para o teste de MSSV, 70 (53,85\%) escolares fizeram três acertos, $49(37,69)$ acertaram duas alternativas, 7 (5,38\%) acertaram apenas uma e 4 $(3,08 \%)$ acertaram nenhuma. Apenas uma criança apresentou articulação da fala incorreta para os sons verbais solicitados, sendo esta para a sílaba "ca". Na tabela 3 estão os resultados do teste de MSSV.

Tabela 3 - Resultados do teste de MSSV na amostra estudada

\begin{tabular}{lccccc}
\hline \multirow{2}{*}{ Memória Sequencial } & \multicolumn{3}{c}{ Sons Verbais } & \multirow{2}{*}{ Total } \\
\cline { 2 - 5 } & & Sequência 1 & Sequência 2 & Sequência 3 & \\
\hline \multirow{2}{*}{ Acerto } & $\mathrm{n}$ & 118 & 107 & 91 & 130 \\
& $\%$ & 90,77 & 82,31 & 70,00 & 100,00 \\
\hline
\end{tabular}

No teste de MSSNV, 5 escolares (3,94\%) tiveram nenhum acerto, $18(14,17 \%)$ tiveram um acerto, $53(41,73 \%)$ e $51(40,16 \%)$ obtiveram dois e três acertos, respectivamente. A tabela 4 apresenta os resultados do teste de MSSNV. Das 130 crianças que realizaram a avaliação simplificada de processamento auditivo, $99(76,15 \%)$ passaram e $31(23,85 \%)$ falharam.

Não houve associação significante entre o resultado da triagem imitanciométrica e da avaliação simplificada de processamento auditivo $(p>0,05)$ (tabela 5). 
Tabela 4 - Resultados do teste de MSSNV na amostra estudada

\begin{tabular}{lccccc}
\hline \multirow{2}{*}{ Memória Sequencial } & \multicolumn{3}{c}{ Sons Instrumentais } & \multirow{2}{*}{ Total } \\
\cline { 3 - 5 } & & Sequência A & Sequência B & Sequência C & \\
\hline \multirow{2}{*}{ Acerto } & $\mathrm{n}$ & 96 & 104 & 85 & 130 \\
& $\%$ & 73,85 & 80,00 & 65,38 & 100,00 \\
\hline
\end{tabular}

Tabela 5 - Associação dos resultados à triagem imitanciométrica e de processamento auditivo. (Teste Qui-quadrado)

\begin{tabular}{|c|c|c|c|c|}
\hline & & \multicolumn{2}{|c|}{ Processamento Auditivo } & \multirow{2}{*}{ Total } \\
\hline & & Falhou & Passou & \\
\hline \multirow{2}{*}{ Imitanciometria } & Falhou & 16 & 58 & 74 \\
\hline & Passou & 15 & 41 & 56 \\
\hline \multicolumn{2}{|c|}{ Total } & 31 & 99 & 130 \\
\hline
\end{tabular}

$(\mathrm{p}=0,634)$

Com relação à idade da amostra e o resultado das avaliações realizadas, constatou-se que a associação da idade com o resultado da triagem de processamento auditivo, assim como com o resultado da triagem imitanciométrica não foi significante $(p>0,05)$ (tabelas 6 e 7$)$.

Tabela 6 - Associação entre resultado da avaliação simplificada de processamento auditivo e idade. (Teste Qui-quadrado)

\begin{tabular}{|c|c|c|c|c|c|c|}
\hline & & \multicolumn{4}{|c|}{ Idade } & \multirow{2}{*}{ Total } \\
\hline & & 7 & 8 & 9 & 10 & \\
\hline \multirow{2}{*}{$\begin{array}{l}\text { Triagem Proces. } \\
\text { Auditivo }\end{array}$} & Falhou & 9 & 6 & 5 & 11 & 31 \\
\hline & Passou & 28 & 18 & 23 & 30 & 99 \\
\hline \multicolumn{2}{|l|}{ Total } & 37 & 24 & 28 & 41 & 130 \\
\hline
\end{tabular}

$(\mathrm{p}=0,855)$

Tabela 7 - Associação entre resultado da triagem imitanciométrica e idade. (Teste Qui-quadrado)

\begin{tabular}{rlccccc}
\hline & & \multicolumn{3}{c}{ Idade } & \multirow{2}{*}{ Total } \\
\cline { 3 - 6 } & & $\mathbf{7}$ & $\mathbf{8}$ & $\mathbf{9}$ & $\mathbf{1 0}$ & \\
\hline Triagem & Falhou & 21 & 13 & 16 & 24 & 74 \\
imitanciométrica & Passou & 16 & 11 & 12 & 17 & 56 \\
\hline \multicolumn{1}{r}{ Total } & & 37 & 24 & 28 & 41 & 130 \\
\hline
\end{tabular}

$(p=0,944)$ 


\section{DISCUSSÃO}

O objetivo deste estudo foi verificar os achados da triagem imitanciométrica e dos testes da avaliação simplificada do processamento auditivo em escolares, bem como a associação entre eles.

Os critérios de "passa" para a triagem imitanciométrica variam conforme a literatura utilizada. No presente estudo, considerando os critérios adotados, $43,08 \%$ das crianças passaram. Em comparação com estudo semelhante, o qual utilizou a mesma forma de classificação, $60,62 \%$ dos escolares apresentaram condições de orelha média normal $^{3}$. Tais resultados, dizem respeito a uma população diferente à do presente trabalho, o que justificaria a diferença entre os achados.

No que diz respeito às medidas de imitância acústica, este estudo mostrou que $80 \%$ das crianças apresentaram curva timpanométrica tipo A na orelha direita e $76,15 \%$ apresentaram curva timpanométrica tipo A na orelha esquerda, a qual sugere funcionamento normal de orelha média. Em outros estudos, a frequência de curvas tipo $A$ variou entre $54 \%$, e $82 \%, 3,19,20 \mathrm{em}$ amostras de escolares. As curvas que sugerem comprometimento de orelha média - tipos $\mathrm{B}, \mathrm{Ad}$, $\mathrm{As}$ e $\mathrm{C}^{18}$, ocorreram em aproximadamente $20 \%$ das crianças. Destas, a curva tipo $\mathrm{C}$ ocorreu com maior frequência, tanto na orelha direita $(13,08 \%)$ quanto na orelha esquerda $(16,92 \%)$, sendo encontrada em indivíduos com mau funcionamento da tuba auditiva ${ }^{17}$. A curva tipo As foi a segunda mais frequente, estando presente em $6,92 \%$ na orelha direita e $6,15 \%$ na orelha esquerda, podendo ser encontrada em indivíduos portadores de otosclerose ou timpanosclerose ${ }^{17}$. A curva tipo $B$ foi não foi encontrada em nenhuma das crianças estudadas. Esta curva, comumente associada à otite média crônica secretora, indica a presença de fluido na orelha média ${ }^{17}$. Devido à grande incidência de otites na população infantil, esperava-se que a frequência de curvas tipo $B$ fosse maior, como no estudo que encontrou $25 \%$ de frequência de curva tipo B em um total de 284 orelhas analisadas ${ }^{19}$. Porém, em estudo realizado com uma população de 287 escolares de cinco a 10 anos, apenas $0,34 \%$ da população apresentou curva timpanométrica tipo $\mathrm{B}^{3}$, de acordo, portanto, com os resultados encontrados no presente estudo.

$\mathrm{Na}$ pesquisa do reflexo acústico ipsilateral, as frequências de 500, 1000 e $2000 \mathrm{~Hz}$ apresentaram percentual de presença semelhante em ambas as orelhas (em torno de $84 \%$ ). Para a frequência de $4000 \mathrm{~Hz}$, esse percentual caiu para $70 \%$ em média, nas orelhas direita e esquerda. Estes achados corroboram os dados encontrados em outra pesquisa realizada com crianças com e sem alteração de processamento auditivo, na qual foi observada diminuição no número de respostas em $4000 \mathrm{~Hz}$ nos dois grupos estudados ${ }^{21}$. Os músculos da orelha média (tensor do tímpano e estapédio) são ativos não somente na presença de sons intensos, mas também em eventos motores, os quais propiciam a melhora do estado de atenção auditiva, percepção de alterações de intensidade, identificação da origem do som e atenuação dos ruídos de baixas frequências provocados por atividades como mastigação, vocalização e movimentos de cabeça, facilitando a percepção de sons externos concomitantes e de altas frequências ${ }^{22}$. Alguns autores ressaltam a importância da realização da pesquisa do reflexo acústico ipsilateral quando o reflexo acústico contralateral estiver ausente em uma ou mais frequências testadas ao suspeitar-se de Transtorno do Processamento Auditivo (TPA) ${ }^{23}$.

Para interpretar informações acústicas, o indivíduo necessita de um conjunto de habilidades auditivas, as quais constituem o processamento auditivo. Dificuldades de memória, de localizar sons e distração são sinais sugestivos de prejuízo das habilidades auditivas e necessitarão de uma avaliação do processamento auditivo ${ }^{24}$. Os testes de processamento auditivo desta pesquisa foram utilizados em estudos anteriores 3,13,21,25-27.

Das 130 crianças que realizaram os testes de processamento auditivo, 99 (76,15\%) passaram e $31(23,85 \%)$ falharam. Em outro estudo, no qual participaram crianças de cinco a dez anos, o percentual de escolares que passou foi de $55,75 \%{ }^{3}$, resultado inferior ao encontrado neste trabalho. Esta diferença pode ter ocorrido pelo fato de o maior índice de falha estar presente no grupo de crianças entre cinco e seis anos de idade, as quais podem apresentar maior dificuldade na realização dos testes devido ao período de maturação da via auditiva ${ }^{28}$. Além disso, a qualidade da vivência acústica propiciada pelo meio familiar e escolar do indivíduo está diretamente relacionada ao comportamento auditivo da criança frente a estímulos auditivos ${ }^{3}$. Em outro trabalho, $78,3 \%$ dos indivíduos passaram nos testes de processamento auditivo ${ }^{29}$, resultado que concorda com os achados desta pesquisa.

Considerando o teste de LS, $58,46 \%$ dos escolares localizaram corretamente 0 som nas cinco direções solicitadas e $93,11 \%$ passaram neste teste. Em um estudo com indivíduos de oito a treze anos de idade, 95,7\% apresentaram condições de normalidade para o teste de $\mathrm{LS}^{27}$. A direção na qual mais ocorreram erros foi "acima" $(71,54 \%)$, concordando com a metodologia utilizada que espera que o erro - quando presente - esteja nesta direção, "atrás" ou "à frente"8. 
No teste de MSSV 91,5\% dos estudantes passaram. Utilizando o mesmo critério, foi encontrado em estudo o total de $82,6 \%$ de resultados satisfatórios para este teste ${ }^{29}$. A maior incidência de erros para o teste de MSSV ocorreu na sequência 3.

Os resultados obtidos na presente pesquisa indicam que o teste da bateria de processamento auditivo no qual os escolares tiveram pior desempenho foi o de MSSNV, corroborando os achados encontrados em pesquisa que refere memória sequencial como sendo a habilidade auditiva mais frequentemente alterada nos indivíduos ${ }^{30}$. Houve mais erros no teste de MSSNV quando apresentada a sequência $C$. Supõe-se que tal resultado se deva ao fato da sonoridade dos três primeiros instrumentos ser semelhante. Além disso, conforme a ordem desta sequência, não havia o instrumento "coco" como entrecorte. O maior percentual de erros para o teste de MSSNV foi encontrado também em outro estudo que aplicou os testes da avaliação simplificada do processamento auditivo em escolares $^{31}$.

A partir da hipótese de que a privação sensorial causada por alterações de orelha média interferiria diretamente no processamento auditivo temporal, pesquisou-se a associação entre o resultado da avaliação simplificada do processamento auditivo e o resultado da triagem imitanciométrica. Constatou-se que não houve associação significante entre os aspectos considerados nos achados desta pesquisa. Não foram encontradas, na literatura compulsada, pesquisas que associassem essas duas variáveis.

No presente estudo, não houve associação significante entre o resultado da triagem de processamento auditivo e idade $(p=0,855)$, porém em outros trabalhos esta associação foi significante ${ }^{3,25}$, mostrando que as habilidades auditivas desenvolvem-se com o aumento da idade. Comparando a metodologia aplicada neste estudo com as utilizadas nos estudos em que esta associação foi significante, pôde-se perceber algumas diferenças. No primeiro trabalho, os escolares participantes da pesquisa tinham de cinco a dez anos de idade e foram avaliados por meio dos testes que compõem a avaliação simplificada do processamento auditivo $^{3}$. No segundo estudo foram selecionadas crianças de oito a dez anos de idade, as quais passaram por uma bateria de testes que avaliaram o processamento auditivo ${ }^{25}$. 0 presente trabalho utilizou apenas um instrumento para avaliar as habilidades do processamento auditivo e contou com uma população de escolares de sete a dez anos de idade, que apresentaram percentual de passa semelhante em cada uma das idades. Estes fatores podem explicar o fato de não ter havido associação significante entre idade e resultado da triagem de processamento auditivo em comparação aos outros estudos.

\section{CONCLUSÃO}

Não houve associação significante entre o resultado da triagem de processamento auditivo e idade e entre o resultado da triagem imitanciométrica e idade.

Não houve associação significante entre os resultados da triagem imitanciométrica e os achados dos testes que compõem a avaliação simplificada do processamento auditivo.

Apesar dos resultados obtidos neste estudo não terem apresentado associação significante, observou-se que a triagem auditiva é instrumento de grande importância para detectar possíveis alterações auditivas que podem interferir no aprendizado dos escolares. Além disso, a atuação da Fonoaudiologia no ambiente escolar pode ocorrer em atividades de orientação e prevenção, destinadas aos pais, alunos e integrantes da equipe escolar, compartilhando conhecimentos relacionados à saúde e à educação, tão importantes neste contexto.

\section{AGRADECIMENTOS}

À Escola Estadual Anne Frank pela colaboração com esta pesquisa. À equipe do projeto de extensão Ouça Bem pelo auxílio na coleta de dados. Ao estatístico Rodrigo C. P. dos Reis pela análise estatística realizada neste estudo. 


\section{ABSTRACT}

Purpose: to check acoustic immittance screening findings and results of the simplified evaluation of auditory processing in school children. Method: the subjects under this study were students from the 1st to the 4th grade, with ages ranging from seven to ten year-old, from a public school in Porto Alegre. 130 students were evaluated in the immitance screening, which consisted of a tympanometry and an ipsilateral acoustic reflex, and a simplified evaluation of auditory processing. This has also involved the tests of sonorous localization, and verbal and non-verbal sequential memory. Results: in the screening immittance, $43.08 \%$ of the students passed and the type A curve was the most frequent. The acoustic reflex at $4000 \mathrm{~Hz}$ had a lower percentage of appearance when compared with the others. In the tests for simplified evaluation of auditory processing, $76.15 \%$ of the children passed. Moreover, it was observed that the worst performance of the evaluated students was found in the verbal sequential memory test. Conclusion: the type A tympanometric curve was the most frequent in this population. In the simplified evaluation of auditory processing, most subjects passed, obtaining higher frequency of correct answers in the sonorous localization test. No statistical association between the results of the immittance screening and the simplified evaluation of auditory processing was found.

KEYWORDS: Hearing; School Health; Auditory Perception

\section{REFERÊNCIAS}

1. Charone A, Negrão A. A fonoaudiologia na escola. Lato \& Sensu. 2001; 2 (4): 7-9.

2. Maranhão PCS, Pinto SMPC, Pedruzzi CM. Fonoaudiologia e educação infantil: uma parceria necessária. Rev CEFAC. 2009; 11 (1): 59-66.

3. Colella-Santos MF, Bragatto GR, Martins PMF, Dias, AB. Triagem auditiva em escolares de 5 a 10 anos. Rev CEFAC. 2009; 11 (4): 644-53.

4. Saffer M, Silva DB, Peduzzi FD, Ávila F. Otite média crônica secretora: conduta expectante. J Pediatr. 2000; 76 (6): 407-12.

5. Costa SS. Aspectos gerais das otites médias. In: Costa SS, Cruz OLM, Oliveira JAA. Otorrinolaringologia: princípios e prática. $2^{\underline{a}}$ ed. Porto Alegre: ArtMed; 2006. p. 254-341.

6. Weckx LLM. Presença ou ausência de bactérias na otite média com efusão. J Pediatr. 2004; 80 (1): 5-6.

7. Saes SO, Goldberg TBL, Montovani JC. Secreção na orelha média em lactentes: ocorrência, recorrência e aspectos relacionados. J Pediatr. 2005; 81 (2): 133-8.

8. Pereira LD. Processamento Auditivo Central: Abordagem Passo a Passo. In: Pereira LD, Schochat E. Processamento Auditivo Central: manual de avaliação. São Paulo: Lovise; 1997, p. 49-60.

9. Harsten G, Nettelblandt U, Schalén L, Kalm, O e Prellner K. Language development in children with recurrent acute otitis media during the first three years of life. Follow- up study from birth to seven years of age. J Laryngol Otolaryngol. 1993; 107: 407-12.

10. Teele DW, Rosner BA, Klein JO, Chase C, Menyuk, $P$ et al.: Otitis media in infancy and intellectual ability, school achievement, speech, and language at age 7 years. J Infect. Dis. 1990; 162: 685-94.

11. Gravel JS, Wallace IF: Listening and language at 4 years of age: Effects of early otitis media. J Speech Hear Res. 1992; 35: 588-95.

12. Jerger S, Jerger J, Alford BR, Abrams S: Development of speech intelligibility in children with recurrent otitis media. Ear Hear. 1983; 4: 138-45.

13. Ramos CS, Pereira LD. Processamento auditivo e audiometria de altas frequências em escolares de São Paulo. Pró-Fono Rev. Atual Cient. 2005; 17 (2): 153-64.

14. Maruthy S, Mannarukrishnaiah J. Effect of early onset otitis media on brainstem and cortical auditory processing. Behav Brain Func. 2008; 17 (4): 1-13.

15. Chermak GD. Auditory processing disorder: An overview for the clinician. The Hearing Journal. 2001; 54(7): 10-25.

16. Gravel JS, Roberts JE, Roush J, Grose J, Besing J, Burchinal $\mathrm{M}$ et al. Early Otitis Media with Effusion, Hearing Loss, and Auditory Processes at School Age. Ear Hear. 2006; 27: 353-68.

17. Russo ICP, Valente CHB, Lopes LQ, BrunettoBorginanni LM. Medidas de Imitância Acústica. In: Momensohn-Santos TM, Russo ICP. Prática da Audiologia Clínica. 7ª̣ Ed. São Paulo: Cortez; 2009. p. 183-216. 
18. Jerger S, Jerger J. Alterações auditivas: um manual para a avaliação clínica. São Paulo: Atheneu; 1989. p. 102.

19. Vasconcelos RM, Monte MO, Aragão VMF, Silva BTF. Alterações auditivas em crianças de 7 a 9 anos de idade de uma escola pública de ensino fundamental em São Luis, Maranhão. Rev Bras Prom Saúde. 2007; 20 (3): 155-60.

20. Guida HL, Diniz TH. Perfil audiológico em crianças de 5 a 10 anos de idade. Arq Int Otorrinolaringol. 2008; 12 (2): 224-9.

21. Linares $A E$, Carvallo RMM. Latência do Reflexo Acústico em crianças com alteração do processamento auditivo. Rev Arq Int Otorrinolaringol. 2004; 8 (1): 11-8.

22. Simmons FB. Perceptual theories of middle ear muscle function. J Acoust Soc.; 1962; 34: 1524-34.

23. Marotta RMB, Quintero SM e Marone SAM. Estudo Comparativo entre os Achados dos Testes SSW e Reflexo Acústico em Indivíduos Adultos com Audição Normal. Revista Arq Int Otorrinolaringol. 2002; 6(2): 104-12. Disponível em: http://www. arquivosdeorl.org.br/conteudo/acervo_port. asp? $\mathrm{id}=187$

24. Pereira LD. Avaliação do Processamento Auditivo Central. In: Lopes Filho OC. Tratado de Fonoaudiologia. $1^{\mathrm{a}}$ ed. São Paulo: Rocca, 1997.
25. Neves IF, Schochat E. Maturação do processamento auditivo em crianças com e sem dificuldades escolares. Pró-Fono Rev. Atual Cient. 2005; 17 (3): 311-20.

26. Elias KMIF, Colella-Santos MF, Ciasca SM, Moura-Ribeiro MVL. Processamento auditivo em criança com doença cerebrovascular. Pró-Fono R. Atual. Cient. 2007; 19 (4): 393-400.

27. Santos JN, Lemos SMA, Rates SPM, Lamounier JA. Habilidades auditivas e desenvolvimento de linguagem em crianças. Pró-Fono Rev. Atual Cient. 2008; 20 (4): 255-60.

28. Simon LF, Rossi AG. Triagem do processamento auditivo em escolares de 8 a 10 anos. Psicol esc educ. 2006; 10 (2): 293-304.

29. Vale SL. Avaliação simplificada do processamento auditivo em crianças de uma Escola Pública de Belo Horizonte [monografia]. Belo Horizonte (MG): UFMG; 2009.

30. Meneguello J, Domenico MLD, Costa MCM, Leonhardt FD, Babosa LHF, Pereira LD. Ocorrência de reflexo acústico alterado em desordens do processamento auditivo. Rev Bras Otorrinolaringol. 2001; 67(6): 830-5.

31. Furbeta TDL, Felippe ACN. Avaliação simplificada do processamento auditivo e dificuldades de leitura e escrita. Pró-Fono Rev. Atual Cient. 2005; 17(1):11-8.
http://dx.doi.org/10.1590/S1516-18462012005000028

RECEBIDO EM: 05/05/2011

ACEITO EM: 26/07/2011

Endereço para correspondência:

Mariana Citton Padilha dos Reis

Rua Amapá, 515, Bairro Vila Nova

Porto Alegre - RS

CEP: $91740-460$

E-mail: marianacpdosreis@gmail.com 